\title{
Analysis of beam plasma instability effects on incoherent scatter spectra
}

\author{
M. A. Diaz ${ }^{1, *}$, J. L. Semeter ${ }^{2}$, M. Oppenheim ${ }^{3}$, and M. Zettergren ${ }^{4, *}$ \\ ${ }^{1}$ Electrical Engineering Department, University of Chile, Santiago, Chile \\ ${ }^{2}$ Electrical and Computer Engineering Department and Center for Space Physics, Boston University, Boston, MA, USA \\ ${ }^{3}$ Astronomy Department, Boston University, Boston, MA, USA \\ ${ }^{4}$ Physical Sciences Department, Embry-Riddle Aeronautical University, Daytona Beach, FL, USA \\ *formerly at: Electrical and Computer Engineering Department and Center for Space Physics, Boston University, Boston, \\ MA, USA
}

Received: 14 July 2010 - Revised: 29 October 2010 - Accepted: 4 November 2010 - Published: 10 December 2010

\begin{abstract}
Naturally Enhanced Ion Acoustic Lines (NEIALs) detected with Incoherent Scatter Radars (ISRs) can be produced by a Langmuir decay mechanism, triggered by a bump on tail instability. A recent model of the beam-plasma instability suggests that weak-warm beams, such those associated with NEIAL events, might produce Langmuir harmonics which could be detected by a properly configured ISR. The analysis performed in this work shows that such a beamdriven wave may be simultaneously detected with NEIALs within the baseband signal of a single ISR. The analysis shows that simultaneous detection of NEIALs and the first Langmuir harmonic is more likely than simultaneous detection of NEIALs and enhanced plasma line. This detection not only would help to discriminate between current NEIAL models, but could also aid in the parameter estimation of soft precipitating electrons.
\end{abstract}

Keywords. Ionosphere (Ionospheric irregularities)

\section{Introduction}

Incoherent Scatter Radar (ISR) is a powerful technique for probing the Earth's ionosphere and its interactions with the space environment. ISRs operate well above the critical frequency of the ionosphere and measure the Thomson Scatter from ionospheric electrons (Evans, 1969). The Doppler spectrum of the received signal reveals information about both electron and ion dynamics due to the strong interactions

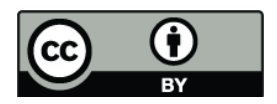

Correspondence to: M. A. Diaz (mdiazq@ing.uchile.cl) between these species. Any wave mode in the plasma that satisfies the Bragg conditions for the given radar frequency, where $\boldsymbol{k}_{\text {Bragg }}=2 \boldsymbol{k}_{\text {radar }}$ for back scattering, will contribute to the scattered signal. The majority of the back scattered power is confined within a narrow (typically tens of $\mathrm{kHz}$ ) double humped band centered at the transmitted frequency. This is referred to as the ion line, or ion spectrum, and its shape is determined by ionospheric parameters such as the electron to ion temperature ratio, ion drift velocity, and electron density. The utility of ISR lies in the ability to estimate these state parameters from measurements of the ion line using inversion techniques (Lehtinen and Huuskonen, 1996). A second feature commonly detected by ISR is the Langmuir mode, which manifests as narrow bands offset from the carrier by the mean plasma frequency of the backscatter volume. Although much weaker in general, this feature has also been used to derive parameters of the ionospheric plasma, such as the critical frequency (Showen, 1979), the density of suprathermal electrons (Nilsson et al., 1997), and the ambient electron temperature (Nicolls et al., 2006).

Embedded in the standard ISR inversion technique is the assumption that the plasma is stationary, homogeneous and Maxwellian within the scattering volume and radar integration period. At high latitude, in the polar cusp and the auroral zone, these assumptions can be violated due to particle precipitation and the presence of localized parallel electric fields in the topside ionosphere (Maurice et al., 1996). One manifestation of these departures from a "well-behaved" plasma is the so called Naturally Enhanced Ion Acoustic Line (NEIAL), often observed in high latitudes ISR data. NEIALs occur during intense red aurora and soft electron precipitation. Enhancements in the spectrum are generally

Published by Copernicus Publications on behalf of the European Geosciences Union. 
2 to 3 orders of magnitude larger than the spectrum of a Maxwellian plasma, NEIALs appear between altitude ranges from $200 \mathrm{~km}$ to $1500 \mathrm{~km}$, the upshifted and downshifted spectral lines can be enhanced simultaneously, NEIALs are more frequently detected at lower radar frequencies, such as $224 \mathrm{MHz}$, than at higher frequencies, such as $930 \mathrm{MHz}$, in fact, no NEIAL has been detected at $1290 \mathrm{MHz}$ (SedgemoreSchulthess and St Maurice, 2001, and references therein).

Several theories have emerged in order to explain the source of NEIALs. The ion acoustic line can be enhanced by two stream instabilities such as the ion-ion two stream instability, causing de-stabilization of ion acoustic waves due to counter-streaming ion populations (e.g. Wahlund et al., 1992), or the current driven instability, resulting from a counter-streaming thermal ion and electron population (e.g. Foster et al., 1988; Rietveld et al., 1991). Another different mechanism to enhance the ion acoustic line is proposed by Forme (1993), where through a two step wave-wave interaction process a beam driven instability generates growing Langmuir waves that can decay into ion acoustic waves and opposite propagating Langmuir waves. A more recent theory proposed by Bahcivan and Cosgrove (2008) postulates that ion acoustic waves can be destabilized through a twostep process, where Electrostatic Ion Cyclotron (EIC) waves can produce non-Maxwellian electron distribution function, which creates inverse Landau damping (assuming the ions are Maxwellian). Only the Langmuir decay (Forme, 1993) and the EIC-electron distribution interaction (Bahcivan and Cosgrove, 2008) models can explain the simultaneous enhancement of both shoulders in the ion acoustic line.

Recent developments in observations and theory seem to discard the two-stream explanations. Grydeland et al. (2003) presented high time resolution data from the EISCAT Svalbar Radar (ESR), from which they conclude that the two shoulders of the ion line are in fact enhanced simultaneously, and not the results of temporal averaging as suggested by Rietveld et al. (1991), and that a temporal resolution of $\sim 0.2 \mathrm{~s}$ is sufficient to resolve the dynamics of the NEIAL. Michell et al. (2009) confirm this result with a temporal resolution of $19 \mathrm{~ms}$. They found that although enhancement of both shoulders in the ion acoustic line is less frequent, it happens within this time resolution. The fast changes in the enhancement from the up to down shoulder measured in the ion acoustic line (Michell et al., 2009) also support the Forme (1993) and Bahcivan and Cosgrove (2008) models. Blixt et al. (2005) collected all the datasets that included both NEIAL and conjugate high-resolution narrow angle optical images of the aurora. Using these data, they suggested that dynamic rayed aurora were causally connected with the formation of the NEIAL. This type of aurora is associated with Alfvénic acceleration of electrons, which produces large numbers of low energy (sub-keV) precipitating electrons as required by the nonlinear decay of Langmuir waves (Forme, 1993, 1999). Michell et al. (2009) also used images of aurora to conclude that dynamic aurora is related to NEIALs, which also are connected to Alfvén waves. Strømme et al. (2005) presented ESR observations of simultaneously enhanced ion and plasma lines. Of the aforementioned theories, only the Langmuir turbulence model (Forme, 1993) can account for the simultaneous enhancement in both shoulders of the ion line and the simultaneous enhancement of ion and plasma lines. However, the work by Strømme et al. (2005) is not conclusive about the correlation between the simultaneous enhancement of plasma and ion lines, since enhancement of both lines may still be caused independently.

The purpose of this paper is to provide, based on plasma physics results, a unified theoretical model of modes expected in the ISR spectrum in the presence of field-aligned electron beams, and to specify the constraints under which these modes may be detected by a single-frequency ISR. The work is motivated by the phenomenological studies summarized above, in addition to recent theoretical results, in particular, those of Yoon et al. (2003), and references therein, which showed that Langmuir harmonics arise as a natural consequence of low-energy, beam-driven, electron-ion instabilities - the same conditions thought to produce NEIALs. Although these effects have been treated in considerable detail in the plasma physics literature, their implications for the field of ionospheric radio science (and ISR in particular) have not yet been discussed.

\section{Plasma in thermal equilibrium}

There exists two natural electrostatic longitudinal modes in an unmagnetized plasma in thermal equilibrium: the ion acoustic mode, which is the main mode detected by ISRs, and the Langmuir mode. Solving the linearized VlasovPoisson system of equations the dispersion relation of these modes is obtained. The real part of the ion acoustic dispersion relation is

$\omega_{\mathrm{s}}^{2}=C_{\mathrm{s}}^{2} k^{2}$,

and the imaginary part (assuming $\omega_{\mathrm{si}} \ll \omega_{\mathrm{s}}, k^{2} \lambda_{\text {De }}^{2} \ll 1$ and $\left.T_{\mathrm{i}} / T_{\mathrm{e}} \ll 1\right)$ can be written as

$\omega_{\mathrm{si}}=-\sqrt{\frac{\pi}{8}}\left[\left(\frac{m_{\mathrm{e}}}{m_{\mathrm{i}}}\right)^{\frac{1}{2}}+\left(\frac{T_{\mathrm{e}}}{T_{\mathrm{i}}}\right)^{\frac{3}{2}} \exp \left(-\frac{T_{\mathrm{e}}}{2 T_{\mathrm{i}}}-\frac{3}{2}\right)\right] \omega_{\mathrm{s}}$

where $C_{\mathrm{S}}=\sqrt{k_{\mathrm{B}}\left(T_{\mathrm{e}}+3 T_{\mathrm{i}}\right) / m_{\mathrm{i}}}$ is the ion-acoustic speed (Boyd and Sanderson, 2003). The dependence of this mode on ionospheric state parameters is observed in Eq. (2). The Langmuir mode is detected by ISR under certain conditions and the real part of its dispersion relation is expressed as

$\omega_{\mathrm{L}}=\sqrt{\omega_{\mathrm{pe}}^{2}+3 k^{2} v_{\text {the }}^{2}} \approx \pm\left(\omega_{\mathrm{pe}}+\frac{3}{2} v_{\text {the }} \lambda_{\mathrm{De}} k^{2}\right)$,

and the imaginary part (assuming $\omega_{\mathrm{Li}} \ll \omega_{\mathrm{L}}$ ) is

$\omega_{\mathrm{Li}} \approx-\sqrt{\frac{\pi}{8}} \frac{\omega_{\mathrm{pe}}^{3}}{k^{3}} \frac{1}{v_{\text {the }}^{3}} \exp \left(-\frac{\omega_{\mathrm{pe}}^{2}}{2 k^{2} v_{\text {the }}^{2}}-\frac{3}{2}\right) \omega_{\mathrm{L}}$. 
The forward model used to estimate ionospheric parameters in ISR assumes that these are the dominating modes in the ISR spectrum. However, an injected beam of particles, in particular electrons, can destabilize the plasma, altering the dispersion relation and amplitude of these modes.

\section{Current model of the Langmuir decay process for NEIAL formation}

The model presented by Forme (1993) to explain NEIALs is a two-step process. First, a beam-plasma instability enhances Langmuir Waves (LWs). Second, if the enhancement of LWs is high enough then the enhanced LWs can decay, enhancing Ion Acoustic Waves (IAWs) and counterpropagating LWs. The plasma-beam process involves three species: thermal electrons, thermal ions, and an electron beam with a bulk velocity. By assuming small perturbations and small damping/growth $\left(v_{\text {the }}, v_{\text {thi }} \ll \omega / k\right)$, linearization of Vlasov-Poisson system can be used to find the dispersion relation of the modes present in the plasma, namely the ion acoustic, Langmuir and beam modes (Boyd and Sanderson, 2003). The beam mode has a dispersion relation given by

$\omega_{\mathrm{b}}=v_{\mathrm{b}} k$,

where $v_{\mathrm{b}}$ is the bulk velocity of the beam. The Langmuir mode, including the beam effect, has a dispersion relation for the real part that is approximately equal to that presented in Eq. (3) and for the imaginary part (Guio and Forme, 2006),

$\gamma_{\mathrm{b}}=-\sqrt{\frac{\pi}{8}}\left(\frac{\omega_{\mathrm{pe}}}{k \Delta v_{\mathrm{b}}}\right)^{2} \omega_{\mathrm{L}} \frac{n_{\mathrm{b}}}{n_{0}} \frac{\omega_{\mathrm{L}}-k v_{\mathrm{b}}}{k \Delta v_{\mathrm{b}}} \exp \left(-\left[\frac{\omega_{\mathrm{L}}-k v_{\mathrm{b}}}{\sqrt{2} k \Delta v_{\mathrm{b}}}\right]^{2}\right)$.

where $\Delta v_{\mathrm{b}}$ is the beam thermal speed.

The total positive complex part, or total growth rate, is obtained by adding Eqs. (6) and (4).

$\omega_{\mathrm{bi}}=\omega_{\mathrm{Li}}+\gamma_{\mathrm{b}}$.

The instability arises when the positive value of $\gamma_{b}$ overcomes the negative values from Landau damping.

Using quasi-linear theory and the quasi-linear relaxation approximation, where the variation with time of the electrostatic energy $W$ is assumed to be equal to the negative value of the total growth rate $\omega_{\mathrm{bi}}$ times the energy $W$

$\partial W(v) / \partial t=-\omega_{\mathrm{bi}}(v) W(v)$,

it is possible to find the time-asymptotic energy in the electron beam instability (Melrose, 1986),

$W_{\mathrm{L}}=\frac{1}{2} \epsilon_{0} E_{\mathrm{L}}^{2} \approx \frac{m_{\mathrm{e}} n_{\mathrm{b}}}{v_{\mathrm{b}}-\Delta v_{\mathrm{b}}}\left[\frac{1}{3}\left(v_{\mathrm{b}}^{3}-\Delta v_{\mathrm{b}}^{3}\right)-\frac{\Delta v_{\mathrm{b}}}{2}\left(v_{\mathrm{b}}^{2}-\Delta v_{\mathrm{b}}^{2}\right)\right]$,

where $\Delta v_{\mathrm{b}}<v_{\mathrm{b}}$ is assumed. When $\Delta v_{\mathrm{b}}$ converges to 0 , the energy gained by the LW converges to two-thirds of the beam energy $\left(1 / 2 m_{\mathrm{e}} n_{\mathrm{b}} v_{\mathrm{b}}^{2}\right)$, while the remainder remains in the beam. Hence, the increment of the LW amplitude $\left(E_{\mathrm{L}}\right)$ due to the interaction of the $\mathrm{LW}$ with the electron beam is expressed as

$E_{\mathrm{L}}^{2} \approx \frac{2}{3} \frac{m_{\mathrm{e}} n_{\mathrm{b}} v_{\mathrm{b}}^{2}}{\epsilon_{0}}$.

If the beam velocity, $v_{\mathrm{b}}$, is large enough to make Eq. (5) intersect the Langmuir dispersion equation at a low wavenumber, making the intersection frequency very close to the plasma frequency $w_{\text {pe }}$, then the wavenumber of the excited Langmuir wave L0 is

$k_{\mathrm{L} 0} \approx \frac{\omega_{\mathrm{pe}}}{v_{\mathrm{b}}}$.

The destabilization of L0 does not guarantee decay into counter-propagating Langmuir (L1) and ion acoustic (s1) waves. To produce this decay, the electric field amplitude of L0, given by Eq. (10), has to overcome a threshold $E_{\text {thr }}$, which is obtained by equating the power transferred from one high frequency wave to another (Fejer, 1979),

$E_{\mathrm{L}}^{2}>\underbrace{\frac{2 n_{\mathrm{i}} k_{\mathrm{B}} T_{\mathrm{i}}}{\epsilon_{0}} \frac{v}{\omega_{\mathrm{pe}}} B_{\max }^{-1}}_{E_{\mathrm{thr}}^{2}}$.

where $v$ represents the ion collision frequency, $\omega_{0}=\omega_{\text {pe }}$ was assumed and $B_{\max } \sim 0.58$ is the maximum value of the function

$B=\frac{T_{\mathrm{i}}^{2}}{T_{\mathrm{e}}^{2}} \Im\left(\frac{T_{\mathrm{i}}}{T_{\mathrm{e}}}+1-2 \alpha_{i} e^{-\alpha_{i}^{2}} \int_{0}^{\alpha_{i}} e^{\tau^{2}} d \tau-i \sqrt{\pi} \alpha_{\mathrm{i}} e^{\alpha_{\mathrm{i}}^{2}}\right)^{-1}$,

obtained for most ionospheric conditions, where $\Im()$ represents the imaginary part and $\alpha_{\mathrm{i}}=(\omega / k) \sqrt{m_{\mathrm{i}} / 2 k_{\mathrm{B}} T_{\mathrm{i}}} . B_{\max }$ is reached when $\omega / k=C_{\mathrm{s}}$.

The connection between the dispersion relation of the different waves produced in this decay process is obtained by imposing conservation of energy and momentum. Hence,

$\omega_{\mathrm{L} j}=\omega_{\mathrm{L} j+1}+\omega_{\mathrm{s} j+1}^{*}$

$\boldsymbol{k}_{\mathrm{L} j}=\boldsymbol{k}_{\mathrm{L} j+1}+\boldsymbol{k}_{\mathrm{s} j+1}$

where $j=0,1,2, \ldots$, and the superscript $*$ stands for the complex conjugate of the complex frequency.

Equations (13) and (14) can be used in combination with Eqs. (1) and (3) as described in Fig. 1 to obtain the enhanced wavenumbers $k_{\mathrm{L} 1}, k_{\mathrm{s} 1}, k_{\mathrm{L} 2}$ and $k_{\mathrm{s} 2}$ in terms of the wavenumber $k_{\mathrm{L} 0}$ of the originally beam-enhanced LW. Weak turbulence theory assumes the linear modes remain valid at all times. Thus, the upper solid line in Fig. 1 shows the dispersion relation of the electron Langmuir oscillations, given by Eq. (3), and the lower trace in Fig. 1 displays the ion-acoustic mode, with a dispersion relation given by Eq. (1).

Inserting Eqs. (1) and (3) into Eq. (13) for $j=0$ yields

$k_{\mathrm{L} 0}^{2}-k_{\mathrm{L} 1}^{2}=\left(k_{\mathrm{L} 0}-k_{\mathrm{L} 1}\right)\left(k_{\mathrm{L} 0}+k_{\mathrm{L} 1}\right)=k_{0} k_{\mathrm{s} 1}$, 


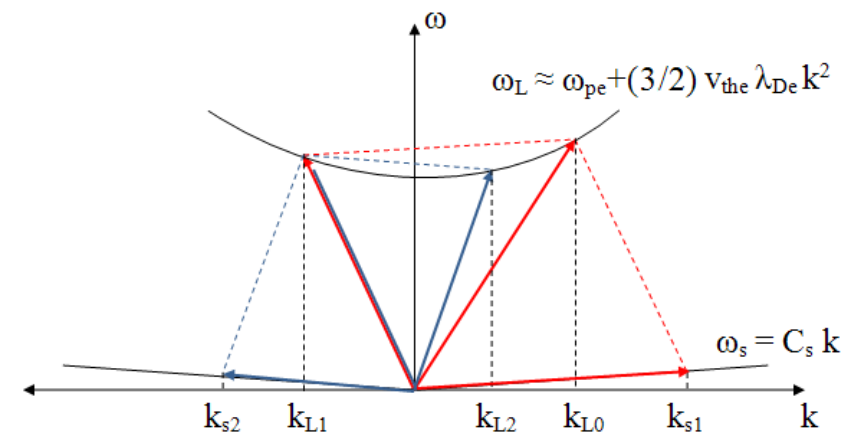

Fig. 1. Simplified schematic of the parametric decay.

where $k_{0}=2 C_{\mathrm{s}} / 3 v_{\text {the }} \lambda_{\text {De }}$. Combining Eq. (14) for $j=0$ with Eq. (15) gives $k_{\mathrm{L} 1}$ and $k_{\mathrm{s} 1}$ in terms of $k_{\mathrm{L} 0}$.

$k_{\mathrm{L} 1}=k_{0}-k_{\mathrm{L} 0}$

$k_{\mathrm{s} 1}=2 k_{\mathrm{L} 0}-k_{0}$.

If the enhancement of the secondary LW L1 is strong enough, it might decay into another LW L2 and an IAW s2 $(\mathrm{L} 1 \rightarrow \mathrm{L} 2+\mathrm{s} 2)$ whose wavenumbers $k_{\mathrm{L} 2}$ and $k_{\mathrm{s} 2}$ can be calculated in terms of $k_{\mathrm{L} 1}$ using a similar procedure. If $k_{\mathrm{L} 0}$ is assumed positive, the wavenumber of the source LW is negative, $k_{\mathrm{L} 1}<0$, making $k_{\mathrm{s} 2}$ and $\omega_{\mathrm{s} 2}\left(-k_{\mathrm{s} 2} C_{\mathrm{s}}\right)$ also negative (Fig. 1). Thus,

$k_{\mathrm{L} 2}=-k_{0}-k_{\mathrm{L} 1}$

$k_{\mathrm{s} 2}=2 k_{\mathrm{L} 1}+k_{0}$,

which can be written in terms of the wavenumber of the original excited Langmuir wave $k_{\mathrm{L} 0}$ by using Eq. (16),

$k_{\mathrm{L} 2}=k_{\mathrm{L} 0}-2 k_{0}$

$k_{\mathrm{s} 2}=-2 k_{\mathrm{L} 0}+3 k_{0}$.

\section{Other NEIAL signatures related to large-growth non- linear beam instability}

The beam instability itself might offer new ways to use ISR to estimate the plasma and beam parameters. Usually in a beam interaction with a background plasma, especially those cases where NEIALs might be produced, the slow growth $\left(v_{\text {the }}, v_{\text {thi }}, v_{\mathrm{b}} \ll \omega / k\right)$ and linearity assumptions can be violated. The impact of the slow growth assumption on the dispersion relation was first treated by Bohm and Gross (1949) and Briggs (1964) who found that the dielectric response of the beam has two different limiting forms corresponding to the reactive and kinetic instabilities. O'Neil and Malmberg (1968) distinguished two versions of the instability corresponding to the dispersive character of the growing waves: the waves might be beam modes with $\omega_{\mathrm{r}} \sim v_{\mathrm{b}} k$, or might lie on a mode with $\omega_{\mathrm{r}} \sim \omega_{\mathrm{pe}}$. Gary (1985) numerically verified the existence of the connected Langmuir-beam mode, predicted by O'Neil and Malmberg (1968), and found a criteria to distinguish both modes. Defining,

$\Gamma_{\mathrm{b}}=\left[\frac{v_{\mathrm{b}}}{\Delta v_{\mathrm{b}}}\right]^{3}\left[\frac{n_{\mathrm{b}}}{n_{\mathrm{e}}}\right]$,

the Langmuir beam instability is produced if $\Gamma_{\mathrm{b}}<1$. On the other hand the beam mode instability is produced if $\Gamma_{\mathrm{b}}>1$. Therefore, Eq. (10) is only valid if the Langmuir beam mode is enhanced $\left(\Gamma_{\mathrm{b}}<1\right)$.

The linearity assumption could also be violated in the production of NEIALs. The main effect of the nonlinearity is the appearance of Langmuir harmonics which are not obtained with the previous linear approaches (Apel, 1967; Manheimer, 1971; Joyce et al., 1971; O'Neil and Winfrey, 1972; Kellogg et al., 1986; Schriver et al., 2000). The electrostatic multiple Langmuir Harmonic (LH) waves in the electron beam instability is a known effect previously observed in laboratory (Apel, 1967), in spacecraft observation (Cairns, 1986), in spaceborne rockets (Kellogg et al., 1986), and in various numerical computer simulations (i.e., Joyce et al., 1971; Kasaba et al., 2001). According to these publications, the harmonic waves are excited at multiples of the plasma frequency, $\omega_{\mathrm{Hn}} \sim n \omega_{\text {pe }}$ (where $n=2,3,4, \ldots$ ), and the wave number of the harmonic with frequency in the vicinity of $n \omega_{\text {pe }}$ is given by

$k_{\mathrm{Hn}} \sim n \omega_{\mathrm{pe}} / v_{b}$.

The theories developed in the early 1970s conceived LHs as forced electrostatic perturbations generated by the interaction of trapped electrons in coherent phase-space vortices and large-amplitude coherent Langmuir waves (Drummond et al., 1970; Joyce et al., 1971; Manheimer, 1971; O’Neil et al., 1971; O'Neil and Winfrey, 1972). Since such theories are applicable for a strong-cold beam, and NEIALs are produced by a weak-warm beam, LHs have not yet been conceived as a possible additional signature in ISR spectra containing NEIALs.

Based on more recent simulations, performed by Schriver et al. (2000) and Kasaba et al. (2001), Yoon (2000) proposed that the broadband spectrum and the persistence of the harmonic modes in a fully turbulent stage cannot be discussed solely on the basis of coherent nonlinear dynamics. In this model (Yoon, 2000; Yoon et al., 2003), the harmonics are considered as eigenmodes of a nonlinear plasma system. Yoon et al. (2003) derived the nonlinear dispersion relation for the harmonic Langmuir waves based on a theoretical analysis of the nonlinear plasma spectral balance equation, obtaining

$\omega_{\mathrm{Hn}}=n \omega_{\mathrm{pe}}+\frac{3}{2} k^{2} \lambda_{\mathrm{De}}^{2} \omega_{\mathrm{pe}}-\frac{3[n-1] k_{\mathrm{L} 0}^{2} v_{\mathrm{the}}^{2}}{2 \omega_{\mathrm{pe}}}\left[\frac{k}{k_{\mathrm{L} 0}}-\frac{n}{2}\right]$ 
They were also able to find an analytical expression for the growth rate of $\mathrm{LH}$ waves, given by

$$
\begin{aligned}
\gamma_{\mathrm{Hn}} & =-\sqrt{\pi}\left[\frac{\omega_{\mathrm{Hn}}}{k v_{\text {the }}}\right]^{3}\left[\omega_{\mathrm{Hn}} \exp \left(-\frac{\omega_{\mathrm{Hn}}^{2}}{k^{2} v_{\text {the }}^{2}}\right)\right. \\
& \left.+\frac{n_{\mathrm{b}}}{n_{\mathrm{e}}}\left[\frac{v_{\text {the }}}{\Delta v_{\mathrm{b}}}\right]^{3}\left[\omega_{\mathrm{Hn}}-k v_{\mathrm{b}}\right] \exp \left(-\frac{\left[\omega_{\mathrm{Hn}}-k v_{\mathrm{b}}\right]^{2}}{k^{2} \Delta v_{\mathrm{b}}^{2}}\right)\right] .
\end{aligned}
$$

The work of Yoon et al. (2003) was supplemented by numerical Vlasov simulations made by Umeda et al. (2003) which were used to make a concrete comparison with the theoretical dispersion curves. Gaelzer et al. (2003) formulated and numerically solved the theoretical wave and particle kinetic equations. It was found that under certain initial conditions, one of the solutions represented a power-law-type spectrum for the power intensity of the LH waves.

In light of these results, it is plausible that Langmuir harmonics may be present in weak-warm beams, such as those present in NEIALs formation. The detection of these harmonics, and their inclusion in models of NEIAL formation, might provide a means of quantifying plasma-beam parameters from distorted IS spectra.

\section{Detection of Langmuir harmonic modes with ISR}

Since the electron beam contains a distribution of velocities, the wavenumbers are not discrete values, but rather cover a range of wavenumbers which could allow the simultaneous detection of more than one mode within the baseband spectrum of a single radar. Defining the ranges

$\left[k_{\mathrm{L} 0}^{\min }, k_{\mathrm{L} 0}^{\max }\right]=k_{\mathrm{L} 0} \pm \Delta k_{\mathrm{L} 0}$,

$\left[k_{\mathrm{s} 1}^{\min }, k_{\mathrm{s} 1}^{\max }\right]=k_{\mathrm{s} 1} \pm \Delta k_{\mathrm{s} 1}$ and

$\left[k_{\mathrm{LH} 2}^{\min }, k_{\mathrm{LH} 2}^{\max }\right]=k_{\mathrm{H} 2} \pm \Delta k_{\mathrm{H} 2}$,

and combining them with Eqs. (11), (17) and (20) (for $n=$ 2 ) the wavenumber ranges of the beam excited LW, $\Delta k_{\mathrm{L} 0}$, that ensures the simultaneous detection are found. Figure 2 shows that multiple detection of the LW, L0, and IAW, s1, is possible if

$k_{\mathrm{s} 1}^{\min }<k_{\mathrm{L} 0}^{\max }$

$k_{\mathrm{s} 1}-\Delta k_{\mathrm{s} 1}<k_{\mathrm{L} 0}+\Delta k_{\mathrm{L} 0}$

Using Eq. (17) is possible to express $k_{\mathrm{s} 1}$ in terms of $k_{\mathrm{L} 0}$ as well as approximate $\Delta k_{\mathrm{s} 1}$ to $2 \Delta k_{\mathrm{L} 0}$, giving

$\Delta k_{\mathrm{L} 0}>\left(k_{\mathrm{L} 0}-k_{0}\right) / 3$.

On the other hand, Fig. 3 shows that the first LH, H2, and the IAW, s1, can be simultaneously detected within the same IS radar if

$k_{\mathrm{H} 2}^{\min } \leq k_{\mathrm{s} 1}^{\max }$,

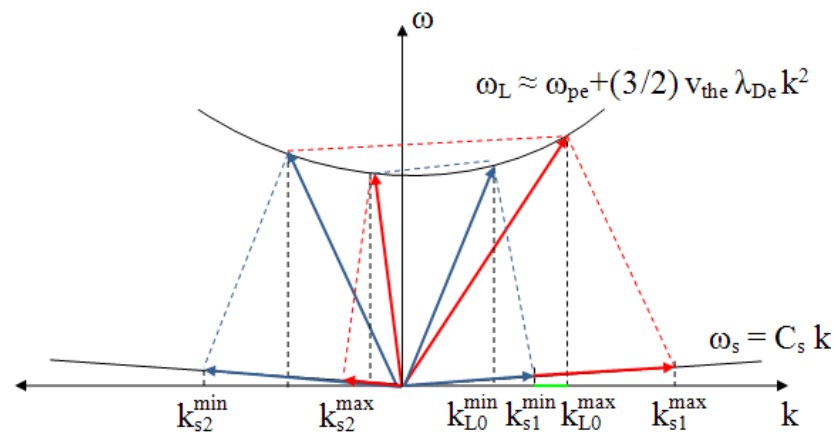

Fig. 2. Schematic of the simultaneous detection of enhanced IAW and LW. The wavenumbers in green would simultaneously detect both enhanced modes.

$k_{\mathrm{H} 2}-\Delta k_{\mathrm{H} 2} \leq k_{\mathrm{s} 1}+\Delta k_{\mathrm{s} 1}$,

Using Eqs. (20) and (11) is possible to express $k_{\mathrm{H} 2}$ in terms of $k_{\mathrm{L} 0}$ as well as approximate $\Delta k_{\mathrm{H} 2}$ by $2 \Delta k_{\mathrm{L} 0}$, hence

$\Delta k_{\mathrm{L} 0} \geq k_{0} / 4$.

Since $k_{0}$, for ionospheric parameters, is around a few $\mathrm{m}^{-1}$ it is possible to conclude from Eqs. (27) and (29) that simultaneous detection of excited IAW-LH requires a narrower wavenumber range of the beam excited LW $\left(\Delta k_{\mathrm{L} 0}\right)$ than that for simultaneous detection of IAW-LW.

It is also possible to find ranges for the velocity dispersion $\Delta v_{\mathrm{b}}$ for simultaneous detection of multiple modes by defining the minimum/maximum wavenumber of the excited LW and the minimum wavenumber of the second $\mathrm{LH}$ as

$k_{\mathrm{L} 0}^{\min }=\frac{\omega_{\mathrm{pe}}}{v_{\mathrm{b}}+\Delta v_{\mathrm{b}}}, \quad k_{\mathrm{L} 0}^{\max }=\frac{\omega_{\mathrm{pe}}}{v_{\mathrm{b}}-\Delta v_{\mathrm{b}}} \quad$ and

$k_{\mathrm{H} 2}^{\min }=\frac{2 \omega_{\mathrm{pe}}}{v_{\mathrm{b}}+\Delta v_{\mathrm{b}}}$,

and combining these definitions again with Eqs. (11), (17) and (20) (for $n=2$ ). Hence, Eq. (26), yields

$2 k_{\mathrm{L} 0}^{\min }-k_{0}<k_{\mathrm{L} 0}^{\max }$

$0<3 \omega_{\mathrm{pe}} \Delta v_{\mathrm{b}}-k_{0}\left(\Delta v_{\mathrm{b}}\right)^{2}-\omega_{\mathrm{pe}} v_{\mathrm{b}}+k_{0} v_{\mathrm{b}}^{2}$.

If $k_{0} \ll 1$ is assumed, the following condition for the thermal speed (or beam width) of the beam is found

$\Delta v_{\mathrm{b}}>(1 / 3) v_{\mathrm{b}}$.

On the other hand, simultaneous detection of IAW and the second LHW is guaranteed if Eq. (28) is met, then

$k_{0} \leq 2 k_{\mathrm{L} 0}^{\max }-2 k_{\mathrm{L} 0}^{\min }$,

$0<k_{0}\left(\Delta v_{\mathrm{b}}\right)^{2}+4 \omega_{\mathrm{pe}} \Delta v_{\mathrm{b}}-k_{0} v_{\mathrm{b}}^{2}$.

which implies $\Delta v_{\mathrm{b}}>0$ if $k_{0} \ll 1$ is assumed. 


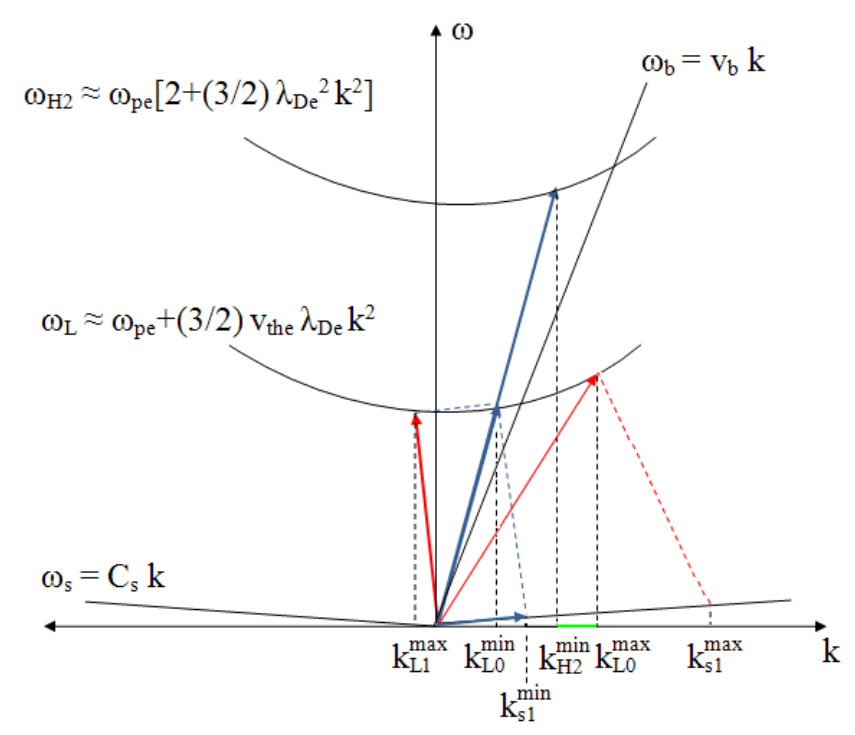

Fig. 3. Schematic of the simultaneous detection of enhanced IAW, LW and the first Langmuir harmonic. The maximum (red colour) and minimum (blue colour) waves follow the definitions presented in Eqs. (30). The decay process of L0 with $k_{\mathrm{L} 0} \in$ $\left[k_{\mathrm{L} 0}^{\min }, k_{\mathrm{L} 0}^{\max }\right]\left(k_{\mathrm{L} 0}^{\min } \rightarrow k_{\mathrm{L} 1}^{\max }+k_{\mathrm{s} 1}^{\min }\right.$ and $\left.k_{\mathrm{L} 0}^{\max } \rightarrow k_{\mathrm{L} 1}^{\min }+k_{\mathrm{s} 1}^{\max }\right)$ locates $k_{\mathrm{s} 1} \in\left[k_{\mathrm{s} 1}^{\min }, k_{\mathrm{s} 1}^{\max }\right]$. The dispersion relation of the beam locates $k_{\mathrm{H} 2} \in\left[2 k_{\mathrm{L} 0}^{\min }, 2 k_{\mathrm{L} 0}^{\max }\right]$. If the condition $v_{\mathrm{b}} \geq 1 / 3 \Delta v_{\mathrm{b}}$ is satisfied, which ensures that $k_{\mathrm{L} 0}^{\min } \leq k_{\mathrm{s} 1}^{\min } \leq k_{\mathrm{H} 2}^{\min } \leq k_{\mathrm{L} 0}^{\max } \leq k_{\mathrm{s} 1}^{\max }$, then multiple detection of the enhanced Langmuir, Ion acoustic and first Langmuir harmonic waves is possible for $k=2 k_{\text {radar }} \in\left[k_{\mathrm{H} 2}^{\min }, k_{\mathrm{L} 0}^{\max }\right]$ (wavenumber interval in green).

There is a $\Delta v_{\mathrm{b}}$ that would allow the detection of the three modes. The LW (L0), the IAW (s1) and the second LHW (LH2) might be simultaneously detected if the condition $k_{\mathrm{H} 2}^{\min }<k_{\mathrm{L} 0}^{\max }$ (see Fig. 3) is met, which yield the condition $\Delta v_{\mathrm{b}}>1 / 3 v_{\mathrm{b}}$ independently of $k_{0}$.

The last case of interest is when both IAW (s1 and s2) are simultaneously enhanced. The condition to satisfy this is $\left|k_{\mathrm{s} 1}^{\min }\right|<\left|k_{\mathrm{s} 2}^{\min }\right|$ (see Fig. 2). By using Eqs. (17) and (19) to express $k_{\mathrm{s} 1}$ and $k_{\mathrm{s} 2}$ in terms of $k_{\mathrm{L} 0}$ in addition to the definitions given by Eq. (30) is possible to obtain

$0<k_{0}\left(\Delta v_{\mathrm{b}}\right)^{2}+\omega_{\mathrm{pe}} \Delta v_{\mathrm{b}}-k_{0} v_{\mathrm{b}}^{2}$.

If $k_{0} \ll 1$ is assumed, the trivial condition $\left(\Delta v_{\mathrm{b}} \geq 0\right)$ for the thermal speed of the beam would guarantee the detection of the enhancement in both shoulders of the IS radar.

The largest beam width found in the previous development, $\Delta v_{\mathrm{b}}>(1 / 3) v_{\mathrm{b}}$ is well within those reported from satellite and rocket measurements, where energy spreads corresponding to $\Delta v_{\mathrm{b}} \in\left[0.5 v_{\mathrm{b}}, 1 v_{\mathrm{b}}\right]$ is commonly measured (Whalen and MacDiarmid, 1972).

\section{Conclusions}

The current explanation of the NEIAL mechanism based on plasma-beam instability and Langmuir decay neglects some important effects. In particular, the condition $\Gamma_{\mathrm{b}}<1$ must be satisfied as a necessary condition for Eq. (12) to be valid, even though this condition is known in plasma physics, it had not been included in the current Langmuir decay-NEIAL model. The expression for $E_{\mathrm{L}}^{2}$ in terms of the beam parameters (Eq. 10) is only valid if $\Gamma_{\mathrm{b}}<1$ is met. In this case, the LWs are actually the waves being enhanced. On the other hand, if $\Gamma_{\mathrm{b}}>1$ is met, the actual mode being enhanced is the beam mode.

A second effect is the appearance of Langmuir harmonics which are also known modes in plasma physics. A recent development of the plasma-beam instability suggests that these harmonics could be developed with weak-warm beams like those present in NEIALs formation, implying that those phenomena might happen simultaneously. The analysis developed in Sect. 5 shows that if both Langmuir harmonics and the NEIAL happen concurrently, it is very likely that the second Langmuir harmonic and IAW enhancement will occur in an overlapping range of wavenumbers, implying that they could be simultaneously detected within the same ISR if the receiver bandwidth of the radar were extended. The constraint found in Sect. 5 for the thermal speed of the beam $\left(\Delta v_{\mathrm{b}}>0\right)$ in order to guarantee simultaneous detection of the second Langmuir harmonic and IAW enhancement suggests that this detection is more likely than for simultaneous detection of IAW and LW $\left(\Delta v_{\mathrm{b}}>1 / 3\right)$. Thus, if the second Langmuir harmonic could be detected with high latitude ISRs, it could help to discriminate between the Langmuir decay-NEIAL and the EIC-NEIAL models. PIC simulation of a Maxwellian plasma injected by a soft electron beam is proposed as future work to evaluate the accuracy of the Langmuir decay-NEIAL model and to study the necessary geophysical characteristics of the beam to develop a second Langmuir harmonic that has similar power than that of the IA line in order to be detected with current IS radars.

Acknowledgements. The authors would like to thank to Topical Editor K. Kauristie and the two anonymous referees for their help in evaluating and improving this paper. This work was supported by the U.S. National Science Foundation under grants AGS 0915386 and AGS 0946611; and an internal grant of Electrical Engineering Department at University of Chile.

Topical Editor K. Kauristie thanks two anonymous referees for their help in evaluating this paper.

\section{References}

Apel, J. R.: Harmonic Generation and Turbulencelike Spectrum in a Beam-Plasma Interaction, Phys. Rev. Lett., 19, 744-746, doi: 10.1103/PhysRevLett.19.744, 1967. 
Bahcivan, H. and Cosgrove, R.: Enhanced ion acoustic lines due to strong ion cyclotron wave fields, Ann. Geophys., 26, 2081-2095, doi:10.5194/angeo-26-2081-2008, 2008.

Blixt, E. M., Grydeland, T., Ivchenko, N., Hagfors, T., La Hoz, C., Lanchester, B. S., Løvhaug, U. P., and Trondsen, T. S.: Dynamic rayed aurora and enhanced ion-acoustic radar echoes, Ann. Geophys., 23, 3-11, doi:10.5194/angeo-23-3-2005, 2005.

Bohm, D. and Gross, E. P.: Theory of Plasma Oscillations. A. Origin of Medium-Like Behavior, Phys. Rev., 75, 1851-1864, doi: 10.1103/PhysRev.75.1851, 1949.

Boyd, T. and Sanderson, J.: The physics of plasmas, Cambridge Univ Pr, 2003.

Briggs, R.: Electron-stream interaction with plasmas, MIT Press, 1964.

Cairns, I. H.: New waves at multiples of the plasma frequency upstream of the earth's bow shock, J. Geophys. Res., 91, 29752988, doi:10.1029/JA091iA03p02975, 1986.

Drummond, W. E., Malmberg, J. H., O’Neil, T. M., and Thompson, J. R.: Nonlinear Development of the Beam-Plasma Instability, Phys. Fluids, 13, 2422-2425, doi:10.1063/1.1693255, 1970.

Evans, J.: Theory and practice of ionosphere study by Thomson scatter radar, Pr. Inst. Electr. Elect., 57, 496-530, 1969.

Fejer, J.: Ionospheric modification and parametric instabilities, Rev. Geophys. Space Phys., 17, 135-153, 1979.

Forme, F.: A new interpretation on the origin of enhanced ion acoustic fluctuations in the upper ionosphere, Geophys. Res. Lett., 20, 2347-2350, 1993.

Foster, J. C., del Pozo, C., Groves, K., and Saint Maurice, J.-P.: Radar observations of the onset of current driven instabilities in the topside ionosphere, Geophys. Res. Lett., 15, 160-163, doi: 10.1029/GL015i002p00160, 1988.

Gaelzer, R., Yoon, P. H., Umeda, T., Omura, Y., and Matsumoto, H.: Harmonic Langmuir waves. II. Turbulence spectrum, Phys. Plasmas, 10, 373-381, 2003.

Gary, S. P.: Electrostatic instabilities in plasmas with two electron components, J. Geophys. Res., 90, 8213-8221, 1985.

Grydeland, T., La Hoz, C., Hagfors, T., Blixt, E., Saito, S., Strømme, A., and Brekke, A.: Interferometric observations of filamentary structures associated with plasma instability in the auroral ionosphere, Geophys. Res. Lett., 30(6), 060000-1, doi: 10.1029/2002GL016362, 2003.

Guio, P. and Forme, F.: Zakharov simulations of Langmuir turbulence: Effects on the ion-acoustic waves in incoherent scattering, Phys. Plasmas, 13, 122902, doi:10.1063/1.2402145, 2006.

Joyce, G., Knorr, G., and Burns, T.: Nonlinear Behavior of the OneDimensional Weak Beam Plasma System, Phys. Fluids, 14, 797801, doi:10.1063/1.1693511, 1971.

Kasaba, Y., Matsumoto, H., and Omura, Y.: One- and twodimensional simulations of electron beam instability: Generation of electrostatic and electromagnetic $2 \mathrm{fp}$ waves, J. Geophys. Res., 106, 18693-18712, 2001.

Kellogg, P. J., Monson, S. J., Bernstein, W., and Whalen, B. A.: Observations of waves generated by electron beams in the ionosphere, J. Geophys. Res., 91, 12065-12077, doi:10.1029/ JA091iA11p12065, 1986.

Lehtinen, M. and Huuskonen, A.: General incoherent scatter analysis and GUISDAP, J. Atmos. Terr. Phys., 58, 435-452, 1996.

Manheimer, W. M.: Strong Turbulence Theory of Nonlinear Stabilization and Harmonic Generation, Phys. Fluids, 14, 579-590, doi:10.1063/1.1693475, 1971

Maurice, J., Kofman, W., and James, D.: In situ generation of intense parallel electric fields in the lower ionosphere, J. Geophys. Res., 101, 335-356, 1996.

Melrose, D.: Instabilities in Space and Laboratory Plasmas, Cambridge University Press, 1986.

Michell, R. G., Lynch, K. A., Heinselman, C. J., and StenbaekNielsen, H. C.: High time resolution PFISR and optical observations of naturally enhanced ion acoustic lines, Ann. Geophys., 27, 1457-1467, doi:10.5194/angeo-27-1457-2009, 2009.

Nicolls, M., Sulzer, M., Aponte, N., Seal, R., Nikoukar, R., and González, S.: High-resolution electron temperature measurements using the plasma line asymmetry, Geophys. Res. Lett., 33, L18107, doi:10.1029/2006GL027222, 2006.

Nilsson, H., Kirkwood, S., Lilensten, J., and Galand, M.: Enhanced incoherent scatter plasma lines, Ann. Geophys., 14, 1462-1472, doi:10.1007/s00585-996-1462-z, 1996.

O’Neil, T. M. and Malmberg, J. H.: Transition of the Dispersion Roots from Beam-Type to Landau-Type Solutions, Phys. Fluids, 11, 1754-1760, doi:10.1063/1.1692190, 1968.

O'Neil, T. M. and Winfrey, J. H.: Nonlinear Interaction of a Small Cold Beam and a Plasma. Part II, Phys. Fluids, 15, 1514-1522, doi:10.1063/1.1694117, 1972

O’Neil, T. M., Winfrey, J. H., and Malmberg, J. H.: Nonlinear Interaction of a Small Cold Beam and a Plasma, Phys. Fluids, 14, 1204-1212, doi:10.1063/1.1693587, 1971.

Rietveld, M., Collis, P., and Saint-Maurice, J.: Naturally enhanced ion-acoustic waves in the auroral ionosphere observed with the EISCAT $933 \mathrm{MHz}$ radar, In Oulu Univ., Fifth EISCAT Scientific Workshop: Programme and Abstracts 1 p (SEE N93-19518 0690), 1991.

Schriver, D., Ashour-Abdalla, M., Sotnikov, V., Hellinger, P., Fiala, V., Bingham, R., and Mangeney, A.: Excitation of electron acoustic waves near the electron plasma frequency and at twice the plasma frequency, J. Goephys. Res., 105, 12919-12928, doi: 10.1029/1999JA000301, 2000.

Sedgemore-Schulthess, F. and St Maurice, J.: Naturally Enhanced Ion-Acoustic Spectra And Their Interpretation, Surv. Geophys., 22, 55-92, 2001.

Showen, R.: The spectral measurement of plasma lines, Radio Sci., 14, 503-508, 1979.

Strømme, A., Belyey, V., Grydeland, T., La Hoz, C., Løvhaug, U., and Isham, B.: Evidence of naturally occurring wave-wave interactions in the polar ionosphere and its relation to naturally enhanced ion acoustic lines, Geophys. Res. Lett., 32, 5, doi: 10.1029/2004GL020239, 2005.

Umeda, T., Omura, Y., Yoon, P. H., Gaelzer, R., and Matsumoto, H.: Harmonic Langmuir waves. III. Vlasov simulation, Phys. Plasmas, 10, 382-391, doi:10.1063/1.1537240, 2003.

Wahlund, J., Forme, F., Opgenoorth, H., Persson, M., Mishin, E., and Volokitin, A.: Scattering of electromagnetic waves from a plasma- Enhanced ion acoustic fluctuations due to ion-ion twostream instabilities, Geophys. Res. Lett., 19, 1919-1922, 1992.

Yoon, P. H.: Generalized weak turbulence theory, Phys. Plasmas, 7, 4858-4871, doi:10.1063/1.1318358, 2000.

Yoon, P. H., Gaelzer, R., Umeda, T., Omura, Y., and Matsumoto, H.: Harmonic Langmuir waves. I. Nonlinear dispersion relation, Phys. Plasmas, 10, 364-372, doi:10.1063/1.1537238, 2003. 